\title{
Non-specific Effect (NSEs) of Supernatant L20B Culture Cell after OPV Exposure against Shigella flexneri Adhesion to Mice Enterocyte
}

\author{
${ }^{1}$ Enny Suswati \\ ${ }^{1}$ Doctoral Program, Faculty of \\ Medicine, Universitas Airlangga \\ Campus A Jl. Prof. Dr. Moestopo 47 \\ $+628123482238$ \\ ennysuswati.fk@unej.ac.id
}

\author{
${ }^{2}$ Fedik Abdul Rantam \\ ${ }^{2}$ Faculty of Veterinary Medicine, \\ Universitas Airlangga \\ Campus C Mulyorejo \\ $+628123593245$ \\ fedik-a-r@fkh.unair.ac.id
}

\author{
${ }^{3}$ Eddy Bagus Wasito \\ ${ }^{3}$ Departement of Clinical Microbiology, \\ Soetomo Teaching Hospital/ Faculty \\ of Medicine, Universitas \\ AirlanggaCampus A Jl. Prof. Dr. \\ Moestopo $47+62$ \\ $+6281330291669$ \\ eddy-b-w@fk.unair.ac.id
}

\begin{abstract}
Introduction Besides protect against specific microorganisms, vaccines can induce heterologous or non-specific effects (NSE). As the global polio eradication initiative prepared to cease use of oral polio vaccine (OPV) in 2020, there is increasing interest in the understanding of oral vaccination provides non-specific immunity to other infections so the consequence of this transition can be adequately planned for and mitigated. Method This research was laboratory experimental research using Randomized Post Test Only Control Group design to prove the difference in adhesion of Shigella flexneri on Balb/c mice enterocytes after variety concentration administration of supernatant L20B Culture cell after OPV exposure. Supernatant concentrations of $0 \%$, $12.5 \%, 25 \%, 50 \%, 100 \%$ each was coated to enterocytes Balb/c mice, then infected with Shigella flexneri. After six hours incubation at $37^{\circ} \mathrm{C}$, a small volume of the mixture was made for Gram staining and examined microscopically under high power field. Shigella flexneri adhere to 100 enterocytes were counted and analyzed using correlation test and Anova. Result here were statistically difference in adhesion between $0 \%$ and $25 \%, 50 \%$ and $100 \% ; 12.5 \%$ and $50 \%$ and $100 \% ; 25 \%$ and $50 \%$ and $100 \% ; 50 \%$ and $100 \%$. There was a strong correlation between supernatant concentration and Shigella flexneri adhesion index in Balb/c mice enterocyte. Conclusion There were index differences of adhesion $S$ flexneri on Balb/c mice enterocyte after giving L20B cell culture supernatant after OPV exposure which was meaningful differences between $0 \%$ with $25 \%, 50 \%$, and $100 \%$. There was strong correlation between supernatant concentration and Shigella flexneri adhesion index in Balb/c mice enterocyte
\end{abstract}

\section{CCS Concepts}

- Applied computing $\rightarrow$ Systems biology

\section{Keywords}

Permission to make digital or hard copies of all or part of this work for personal or classroom use is granted without fee provided that copies are not made or distributed for profit or commercial advantage and that copies bear this notice and the full citation on the first page. Copyrights for components of this work owned by others than ACM must be honored. Abstracting with credit is permitted. To copy otherwise, or republish, to post on servers or to redistribute to lists, requires prior specific permission and/or a fee. Request permissions from Permissions@acm.org.

ICBBS 2019, October 23-25, 2019, Beijing, China

(C) 2019 Association for Computing Machinery.

ACM ISBN 978-1-4503-7251-0/19/10 ...\$15.00

DOI:https://doi.org/10.1145/3369166.3369196
OPV; Shigella flexneri; Adhesion; Non-Specific Effects (NSEs)

\section{INTRODUCTION}

Besides protection against specific microorganisms, vaccines can induce heterologous or non-specific effects (NSE) [1]. As the global polio eradication initiative prepares to cease use of oral polio vaccine (OPV) in 2020, there is increasing interest in understanding if oral vaccination provides non-specific immunity to other infections so that the consequences of this transition can be adequately planned for and mitigated [2].

The family Picornaviridae includes many human and animal pathogens, such as poliovirus, hepatitis A virus, foot-and-mouth disease virus, and rhinovirus. All picornaviruses are small, nonenveloped viruses with a single-stranded RNA genome of positive polarity, properties that reflected in the name of the virus family: pico, a small unit of measurement, and the nucleic acid of the viral genome, RNA. There are three serotypes of poliovirus which classified in the species Enterovirus $\mathrm{C}$ within the genus Enterovirus [3]

Poliovirus (PV) is an enterovirus that is ingested and travels through the gastrointestinal tract where it can initiate infection at several sites, including the tonsils and Peyer's patches of the small intestine. From the initial sites of entry, the virus travels to the draining lymph nodes where it replicates further and spreads via the efferent lymphatic vessels and thoracic duct to enter the bloodstream. In some instances, the virus can spread to the central nervous system (CNS) but rarely (estimated 1 case per 100-200 infections) lead to permanent flaccid paralysis. Infected humans shed poliovirus in the pharyngeal secretions and faeces, usually for 2-8 weeks, implying that virus replicates in the intestine. Presumably, virus contaminates the hands of the infected person and is transmitted by hand to hand contact to the next person in the chain of infection [4].

Classified into three serotypes (PV-1, PV-2, and PV-3), PV belongs to the Picornaviridae family, which is one of the most important groups of human and animal pathogens. This family also includes human hepatitis A virus, human rhinoviruses which is the agents of the common cold, and foot-and-mouth disease virus. Because of its very simple structure, PV has been used as a model for studying non-retroviral RNA viruses, and consequently it is now one of the best characterized animal viruses. The development of new animal and cell models, together with the identification of the virus receptor CD155, has allowed the key steps of the pathogenesis of poliomyelitis to be investigated at the molecular level [3], [4].

Vaccination is based on the induction of specific immunological memory that enhances adaptive immune responses in lymphocytes 
upon subsequent infection with a similar pathogen. Aside from these diseases-specific effects, a growing body of literature is suggesting that widely used vaccines, including the liveattenuated anti-tuberculosis vaccine Bacillus Calmette-Guérin (BCG) and live-attenuated measles vaccine (MV), could induce heterologous protective 'non-specific effects' (NSE), affecting overall mortality [1]. Vaccines against infectious disease undoubtedly have specific disease-protective effects, but there is also increasing evidence that they affect the resistance to other infectious diseases, so-called nonspecific effects, and these effects may be strongly beneficial but also sometimes detrimental [4]

In this study, we will focus on the NSE of Oral Polio Vaccines. (OPV) We will investigate Supernatant L20B Culture Cell after OPV Exposure Against Shigella flexneri Adhesion to Mice Enterocyte.

\section{MATERIAL AND METHOD}

This research was explorative research to prove that L20B Cell Culture produced an immune response cell that was displayed by OPV $[6,7,8,9]$. L20B cells are mouse L cells transfected with the gene for the human cellular receptor for poliovirus. Expression of the receptor at the cell surface renders L20B cells susceptible to infection with poliovirus and develops a typical cytopathic effect. Since the cells are originated from murine, very few other human enteric viruses produce cytopathic infection in L20B cells. This selectivity for polioviruses makes the cell line an attractive option for the network of virological laboratories established by the World Health Organization (WHO) to conduct surveillance for wild polioviruses and to provide evidence for the certification of poliomyelitis eradication. Confluent monolayers of L20B cells in flasks were supplied to participants from a master cell bank of mycoplasma-free cells established at the Regional Government to the Indonesian Ministry of Health's Technical Service Unit, Surabaya, East Java, Indonesia. Participants were requested to subculture the cells every 5-7 days to establish stocks for cryopreservation and for the study. The recommended media for growth and maintenance was bicarbonate-buffered Eagle's modified essential medium (MEM; Earles salt base) supplemented with $10 \% \mathrm{v} / \mathrm{v}$ or $2 \% \mathrm{v} / \mathrm{v}$ newborn (or foetal) calf serum, respectively [6], [8], [10], [11].

The next step is experimental laboratory research in vitro with Randomized Control Group Post Test Only design to prove that there is difference about adhesion S. flexneri on Balb/c mice enterocyte after giving a variety of supernatant concentrations of L20B cells displayed with OPV. The L20B Cell culture Supernatant shown by OPV with concentrations of $0 \%, 12,5 \%$, $25 \%, 50 \%$ dan $100 \%$ is coated to Balb/c mice enterocytes and then is infected with $S$. flexneri, painted with Gram Coloring observed under microscope with large field by counting $S$. flexneri which stickled on 100 enterocytes. Statistic results analyzed with correlation test and Anova.

\section{RESULT}

The L20B cell culture supernatant exposed to OPV with concentrations of $0 \%, 12.5 \%, 25 \%, 50 \%$, and $100 \%$ was coated on $\mathrm{Balb} / \mathrm{c}$ mice enterocyte then mixed with Shigella flexnerri bacterial suspension which is equivalent to $0.5 \mathrm{Mc}$. Farland solution with the same volume comparison. Then preparations were made in the object-glass and Gram stained. Microscopic observations showed that the average amount of $S$. flexneri attached to the Balb / c mice enterocytes that had been coated with supernatant with a concentration of $0 \%$ was less than the concentrations of $12.5 \%, 25 \%, 50 \%$, and $100 \%$ (see Figure 1).

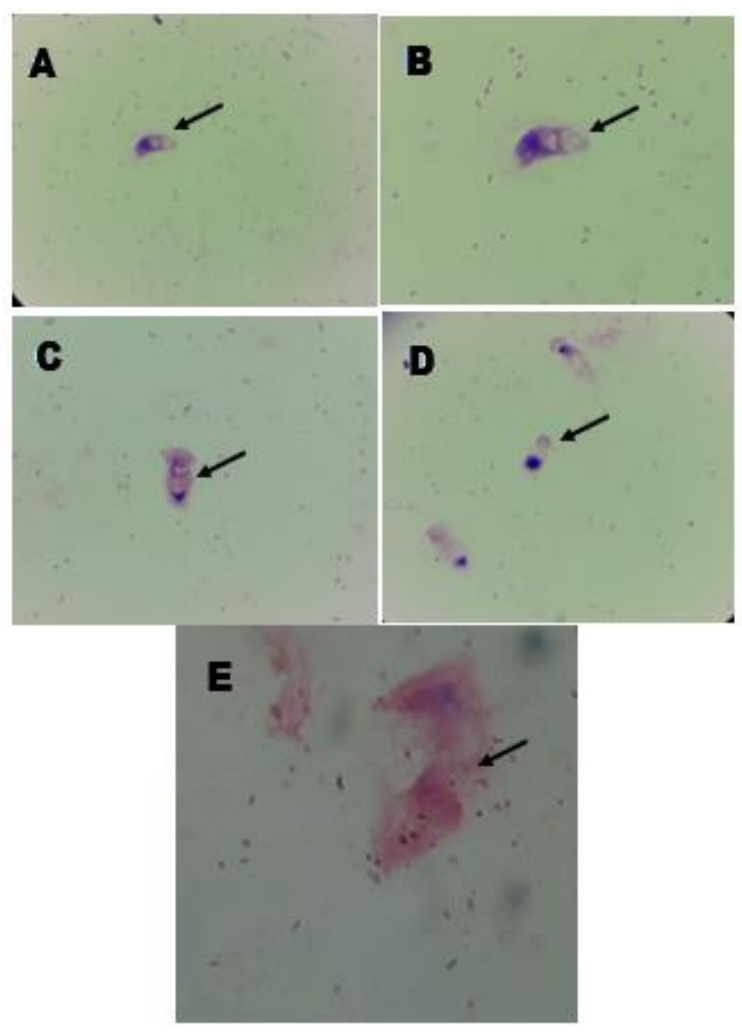

Figure 1. The observation of S. flexneri adhesion on Balb / c mice enterocyte cells after being coated with L20B cell culture supernatant exposed to OPV with various concentrations. (1000x magnification) S. flexneri adhesion to enterocytes after supernatant exposure to concentrations (a) $0 \%$, (b) $12.5 \%$, (c) $25 \%$, (d) $50 \%$, and (e) $100 \%$.

Table 1. Effect of L20B culture supernatant concentrations exposed to OPV to the S. flexneri adhesion index in Balb / c mice enterocytes

\begin{tabular}{|c|c|c|c|c|c|c|}
\hline \multirow{2}{*}{$\begin{array}{c}\text { concentration } \\
\text { of } \\
\text { supernatant }\end{array}$} & \multirow{n}{*}{$\mathbf{n}$} & \multicolumn{4}{|c|}{ Adhesion } & \multirow{2}{*}{$\mathbf{p}$} \\
\cline { 4 - 6 } & & $\overline{\mathbf{x}}$ & SD & Min & Max & \\
\hline $0 \%$ & 6 & $23.21^{\mathrm{a}}$ & 3.70 & 18.30 & 28.00 & \\
\hline $12,5 \%$ & 6 & $41.17^{\mathrm{ab}}$ & 14.11 & 27.00 & 62.00 & \multirow{2}{*}{$0,000^{*}$} \\
\hline $25 \%$ & 6 & $45.36^{\mathrm{b}}$ & 18.71 & 32.20 & 83.00 & \\
\hline $50 \%$ & 6 & $141.38^{\mathrm{c}}$ & 25.36 & 117.33 & 190.00 & \\
\hline $100 \%$ & 6 & $163.27^{\mathrm{d}}$ & 12.28 & 147.33 & 179.66 & \\
\hline
\end{tabular}

* significance at level $\alpha=0,05$ (Oneway Anova) ${ }^{\text {abcd }}$ superscript the difference between group (multiple comparisons LSD)

Oneway ANOVA analysis results showed S. flexneri adhesion index in Balb /c mice enterocytes after administration of L20B cell culture supernatant exposed to OPV treatment group with $0 \%$ concentration was not significantly different from the treatment group $12.5 \%$ concentration. Treatment group after administration of concentration $12,5 \%$ was not significantly different from the treatment group after administration of $25 \%$ concentration but significantly different from the treatment group with a concentration of 50\% and $100 \%$ (see Tab. 1).

The results of the correlation analysis between the concentration of L20B cell culture supernatant exposed to OPV 6 hours postinoculation with adhesion index showed R: 0.927 with $\alpha$ : 0.01 . 
Based on the results of correlation analysis of L20B cell culture supernatants exposed to OPV 6 hours post-inoculation with adhesion index has a very strong correlation.

\section{DISCUSSION}

The results of this study indicate that there are differences in the adhesion index of Balb / $\mathrm{c}$ mice enterocytes after administration of L20B cell culture supernatant exposed to OPV in the treatment group with a concentration of $0 \%$ with concentrations of $25 \%, 50 \%$ and $100 \%$ that are significant $(\mathrm{p}<0.05)$. S. flexneri was able to attach more to enterocytes than the group receiving the supernatant concentration of $0 \%$ and $12.5 \%$.

Previous research stated that there were epidemiological phenomena of non-specific effects (NSEs) or non-related targets by giving several vaccines to newborns. The administration of the BCG vaccine in Sweden since 80 years ago has resulted in a decrease in mortality in general, as well as in Senegal the success of the measles vaccine is followed by an increase in the mortality rate of girls. NSEs from the administration of vaccines have multiple meanings which can be beneficial or otherwise harmful [5].

Other studies have also shown that administration of OPV causes NSEs, namely a decrease in mortality and a decrease in the incidence of diarrhea due to bacterial infections [2]. Epidemiological data show that vaccines have NSE that is more important for children's resistance than their specific effects. Existing studies show that live attenuated vaccines (BCG, Measles, OPV and Vaccinia) are related to NSEs that are beneficial because they reduce mortality with various etiologies whereas DTP vaccines are associated with increased vulnerability of girls to other infections not related to DTP vaccine [13].

Previous studies suggest that infants who get OPV immunization have a lower risk of otitis media poets than babies who get IPV immunization at the age of 6-8 months of life [14]. Likewise raising the mortality of infants aged 4.5 - 36 months decreased with the administration of OPV and BCG during the first two days of birth [12].

Another study stated that administration of IFN- $\gamma$ to ECV304 cells was able to increase adherent group B streptococcus after incubation for 2 hours [13]. The administration of IFN- $\gamma$ was also able to increase the adhesion ability of new isolates of Pseudomonas aeruginosa bacteria in HEp-2 cell cultures [14]. Previous research also stated that TNF- $\alpha$ and IL- $1 \alpha$ plays a role in the pathogenesis of otitis media due to infection with Streptococcus pneumoniae by increasing adherent $S$. pneumoniae in the tubal tympani[15]

The mechanism of bacterial adhesion in cell culture is divided into 3 phases, reversible adhesion, irreversible adhesion, and invasion that are affected by the presence of simultaneous viral infection. Possible because of the change in electrical potential energy in the medium or solution around the cell. The increase in the number of particles in the ocean will reduce the electrical potential energy, thereby increasing the chance of contact between bacteria and host cells. Inoculation of measles viruses that have been attenuated in HEp2 cell culture makes cells more sensitive to secondary bacterial invasion[15].

The adhesion ability of E. coli O157: H7 to HT-29 cells 12 hours earlier exposed to TNF- $\alpha$ decreased, because of TNF- $\alpha$ could increase pro-inflammatory cytokine mRNA secretion (IL-1 $\beta$, IL-8 and TNF- $\alpha$ ). Furthermore, administration of TNF- $\alpha$ will increase
IL-8 production, which is associated with increased phosphorylation of NFp65 [15].

Based on the $S$ flexneri adhesion index test results on Balb / c mice enterocytes that the higher the concentration of L20B cell culture supernatant exposed to OPV the higher the adhesion index, it can be stated that the culture supernatant of L20B cells exposed to OPV has non specific effects that are detrimental to the host because adhesion is higher the first stage of the infection process. this may occur if the supernatant contains compounds that can influence the occurrence of S flexneri adhesion in Balb / c mice enterocytes. This is in accordance with the opinion of Benn et. al that vaccines against infectious disease undoubtedly have specific disease-protective effects but there is also increasing evidence that they affect the resistance to other infectious diseases, so-called nonspecific effects, and these effects may be strongly beneficial but also sometimes detrimental [5].

Oral polio vaccine (OPV) is difficult to study because it is WHO policy to give OPV with DTP. However, it seem that OPV may have beneficial NSE. When OPV was introduced in the 1960s in Chile, a virologist noted that OPV enhanced the interferon-gamma response and reduced the risk of other entero-pathogens and diarrhoeal deaths. If OPV has beneficial NSEs, this has important consequences because most global health researchers want to stop OPV and replace it with inactivated polio vaccine (IPV) [9]. The perceived lack of biological plausibility has been a major obstacle in recognizing and further investigating non-specific effects. Hence, it is important to consider immunological mechanisms that may mediate such effects. Novel insights in understanding both the adaptive immune system and innate immunity has provided arguments thatexposure to a pathogen leads not only to specific immunological memory (represented by memory T- and B-cells), but also to T-cell mediated cross-reactivity, as well as training of the innate immunes

T-cell mediated cross-reactivity heterologous immunity

Each individual has a unique lifelong history of infections and vaccinations, and each exposure leaves an imprint on the immune system that can affect future innate and adaptive immune responses to new pathogens. This conceptof 'heterologous immunity' could explain the observation that vaccines may have non-specific effects, because thevaccines encode antigens that cross-react with other pathogens. In some scenarios, beneficial heterologous immunitycan provide partial protective immunity and be the difference between life and death. In other scenarios, detrimental heterologous immunity can lead to severe immunopathology. Hence, T-cell mediated heterologous immunity provides a plausible biological mechanism by which vaccines may affect the immune response to a subsequent unrelatedinfection and also explains how, in certain situations, avaccine could have detrimental effects on the outcome of secondary infections.

\section{Training of the innate immune system}

Activation of cross-reactive T-cell responses, as seen in heterologous immunity, might explain some of the nonspecific effects of vaccination. However, there is also evidence suggesting that the altered resistance to subsequent infections after vaccination or infection with an unrelated pathogen cannot be attributed to adaptive immune responses alone, and that innate immune responses result in a heightened state of activation. Vaccination of volunteers with BCG showed that in addition to induction of specific T-cell responses, non-specific innate immune responses to unrelated pathogens were also increased for at least 3 months after the vaccination. This 'trained immunity was 
associated in humans with epigenetic reprogramming of monocytes at the level of H3K4 trimethylation. Hence, these data suggest a picture in which the innate immune system is characterized by adaptive features, and can be trained to provide a partial protection against infection independent of the classical Tand B-cell adaptive immunity. These immunological mechanisms do support the biological plausibility by demonstrating that the encounter with one pathogen may alter the immune response to subsequent completely unrelated pathogen challenges, and this may result in improved outcomes, but also at times be detrimental. So far this has been demonstrated most convincingly for BCG [13].

WHO recently reviewed the potential non-specific effects (NSEs) of Bacille Calmette-Guérin vaccine (BCG), diphtheria-tetanuspertussis (DTP), and measles vaccine (MV) $(1,2)$. BCG and MV were associated with large reductions in overall mortality, in the range of halving mortality; these effects were not fully explained by prevention of tuberculosis and measles infection. Hence, the vaccines had "non-specific effects" (NSEs). A growing number of immunological studies supports that vaccines can generate heterologous non-specific protection by inducing cross-reactive Tcells or by training the innate immune system [17].

The current challenge is to determine the appropriate immunological mechanism and can guarantee the existence of similar useful benefits or effects and avoid adverse effects. Furthermore, beneficial effects such as those that occur in Measles Measle and BCG vaccines can make the development of immunization more effective for diseases targeted by vaccines given [18]

\section{CONCLUSION}

Our results suggest that index differences of adhesion $S$ flexneri on Balb/c mice enterocyte after giving L20B cell culture supernatant exposed OPV which was meaningful $0 \%, 12,5 \%$ with $25 \%, 50 \%$, and $100 \%$. There was strong correlation between supernatant concentration and Shigella flexneri adhesion index in $\mathrm{Balb} / \mathrm{c}$ mice enterocyte

\section{REFERENCE}

[1] Bree, LCJ., Koeken, V.A.C.M., Joosten, L.A.B., Aaby, P., Benn, C.S., Crevel, A.V., Netea, M.G. 2018. Non-specific Effects of Vaccines: Current Evidence And potential. Implication Seminar in Immunology Vol.39:35-43

[2] Brwon, A.U., Taniuchi, M., Millis, J.A.P., Kirkpatrick, B., Bargess, S.L., Obersis, M.S., Weidoa, W., Houpt, E., Haque, R., Zaman, K., Petri Jr., W.A. 2017 Nonspecific Effects of Oral polio Vaccine on Diarrheal Burden and Etiology Among Bangladeshi Infants Clinical Infectious Diseases Vol. 65:414-419

[3] Blondel, B., Garapin, F.C., Couderc, T., Wirotius, A., Benhassine, F.G. Poliovirus, Pathogenesis of Poliomyelitis, and Apoptosis CTMI 2005 ; 289:25-56

[4] Nathanson, N. Chapter 1 The Pathogenesis of Poliomyelitis: What We Don't Know Advances in Virus Research 2008 ; Volume 71, 2008, Pages 1-50

[5] Benn, C.S., Netea, M.G., Selin, L.K., Aaby, P. 2013. A small Jab- a Big Effect: Nonspecific Immunomodulation by Vaccines. Trends in Immunology 2013 Sep; Vol. 34 No.9. 431-9. DOI: 10.1016/j.it.2013.04.004

[6] Racaniello, V.R. 2006 One Hundred Years of Poliovirus Pathogenesis, Available online at www.sciencedirect.com Virology 344 (2006) 9 - 16 www.elsevier.com/locate/yviro
[7] Pipkin, P.A., Wood., D.J., Racaniella, V.R., Minor, P.D. Characterization of L Cell Expressing The Human Polio Virus Receptor For The specific Dettection of Poliovirus in Vitro Journal of Virological Methods 1993 ; Volume 41 page: $333-340$

[8] Wood, D.J., Hull, B. L20B Cells Simplify Culture of Polioviruses From Clinical Samples. Journal of Medical Virology. 1999 ; Volume 58 page: 188-192

[9] World Health Organization, 2004, Manual For The Virological Investigation of Poliomyelitis, WHO/EPI/CDS/POLIO/90.1

[10] Kaundal, N., Sarkate, P., Prakash, Rishi, N. 2017 Environmental surveillance of polioviruses with special reference to L20B cell line VirusDis. 28(4):383-389

[11] Chapel, C., Helion, N.R., Mantel, N., Imbert, S., Deshaies, E., Barban, V., Saborraud, A., Barbe, J.P., Mallet, L., 2018, Replacement of Primary Monkey Kidney Cells by L20B Cell Line in the Test for Effective Inactivated Poliovirus Vaccine. Journal of Virological Methods. Volume 256 page:77-84

[12] Aaby, P., Andersen, A., Martins, C.L., Fisker, A.B., Rodrigues, A., Whittie, H.C.,Benn, C.S. 2016 Does Oral Polio Vaccine Have Non-Specifics on All Cause Mortality? Natural Experiments Within a Randomised Controlled Trial of early Measles Vaccine BMJ Open 6:e13335

[13] Sankoh, O., Welaga, P., Debpuur, C., Zandoh, C., Gyaase, S., Poma, M.A., Mutua, M.K., Hanifi, SM. M.A., Martin, C., Nebie, E., Kagone, M., Emina, J. BQ., Aaby, P. 2014 The Non-specific Effects of Vaccines and Other Childhood Interventions the Contribution of INDEPTH Health and Demographic Surveillance Systems International Journal of Epidemiology Editorial 645-653

[14] Seppälä E., Viskari H., Hoppu S., Honkanen H., Huhtala H., Olli Simell O., Ilonen J., Knip M., Hyöty H. 2011 Viral Interference Induced by Live Attenuated Virus Vaccine (OPV) can Prevent Otitis Media. Vaccine Volume 29, Issue 47 Pages 8615-8618

[15] Svanborg, C., Hedlund, M., Connel, H., Agace, W., Duan, R.D., Nilsson, A., Wult, B., 1996 Bacterial Adherence and Mucosal Cytokine Respon, Receptors and Transmembrane SignallingAnn, New York Academic Science Vol. 797. Pp. 177-190

[16] Tong, H.H., Fisher, L.M., kosunick, G.M., Demaria. T.M. 1999 Effect of Tumor Necrosis Factor - $\alpha$ and Interleukin 1$\beta$ on the Adherence of Streptococcus pneumoniae to Chinchilla Tracheal Epithelium Acta Otolaryngol (Stockh); 119: 78-82

[17] A. Andersen, A. B. Fisker ${ }_{1+}$, A. Rodrigues2, C. Martins2, H. Ravn, N Lund, 1 S. B Sørensen 1 , C. S Benn and P. Aaby 2018 National Imunization Campaigns with oral polio Vaccine Reduce Mortality: A Natural Experiment within Seven Randomized Trials Fontier in Public health. www.frontiersin.org vol. 6

[18] Flanagan, K.L, Klein, S.L., Skakebaek, N.E., Marriot, I., Marchant, A., Selin, L., Fish, E.N., Prentice, A.M., Whitte, H., Benn, C.S., Aaby, P. 2011 Sex Differences in the Vaccine-Specific and non-targeted Effects of Vaccines Vaccines; Vol. 29,13: 2349-2354 